This document is confidential and is proprietary to the American Chemical Society and its authors. Do not copy or disclose without written permission. If you have received this item in error, notify the sender and delete all copies.

\title{
Sampled-Current Voltammetry at Microdisk Electrodes: Kinetic Information from Pseudo Steady State Voltammograms
}

\begin{tabular}{|r|l|}
\hline Journal: & Analytical Chemistry \\
\hline Manuscript ID: & ac-2014-02645e.R1 \\
\hline Manuscript Type: & Article \\
\hline Date Submitted by the Author: & n/a \\
\hline Complete List of Authors: & $\begin{array}{l}\text { Perry, Samuel; University of Southampton, Chemistry } \\
\text { Al Shandoudi, Laila; University of Southampton, Chemistry } \\
\text { Denuault, Guy; University of Southampton, School of Chemistry }\end{array}$ \\
\hline
\end{tabular}




\author{
Samuel C. Perry, Laila M. Al Shandoudi, Guy Denuault* \\ Chemistry, University of Southampton, Highfield, Southampton, SO17 1BJ, UK.
}

\begin{abstract}
In sampled-current voltammetry (SCV) current transients acquired after stepping the potential along the redox wave of interest are sampled at a fixed time to produce a sigmoidal current-potential curve akin to a pseudo steady state voltammogram. Repeating the sampling for different times yields a family of sampled-current voltammograms, one for each timescale. The concept has been used to describe the current-time-potential relationship at planar electrodes but rarely employed as an electroanalytical method except in normal pulse voltammetry where the chronoamperograms are sampled once to produce a single voltammogram. Here we combine the unique properties of microdisk electrodes with SCV and report a simple protocol to analyze and compare the microdisk sampled-current voltammograms irrespective of sampling time. This is particularly useful for microelectrodes where cyclic voltammograms change shape as the mass transport regime evolves from planar
\end{abstract}


diffusion at short times to hemispherical diffusion at long times. We also combine microdisk sampled-current voltammetry (MSCV) with a conditioning waveform to produce voltammograms where each data point is recorded with the same electrode history and demonstrate that the waveform is crucial to obtaining reliable sampled-current voltammograms below $100 \mathrm{~ms}$. To facilitate qualitative analysis of the voltammograms we convert the current-potential data recorded at different timescales into a unique sigmoidal curve which clearly highlights kinetic complications. To quantitatively model the MSCVs we derive an analytical expression which accounts for diffusion regime and kinetic parameters. The procedure is validated with the reduction of $\mathrm{Ru}\left(\mathrm{NH}_{3}\right)_{6}{ }^{3+}$, a model one electron outer sphere process, and applied to the derivation of the kinetic parameters for the reduction of $\mathrm{Fe}^{3+}$ on Pt microdisks. The methodology reported here is easily implemented on computer controlled electrochemical workstations as a new electroanalytical method to exploit the unique properties of microelectrodes, in particular at short times.

\section{INTRODUCTION}

Because of its simplicity sampled-current voltammetry (SCV) is a convenient concept often used in textbooks ${ }^{1,2}$ to introduce the theoretical relationship between potential, time and current for simple redox processes at planar electrodes. SCV belongs to the family of pulsed voltammetric methods developed to enhance detection limits and minimize distortion from background processes. ${ }^{3-7}$ In its basic form successive chronoamperograms are recorded after stepping the potential from a rest value where no redox process occurs at an appreciable rate to values where the target Faradaic process takes place. The resulting transients, Figure 1a, are then sampled at different times to yield a family of sampled-current voltammograms (SCVs), each corresponding to a particular sampling time, Figure 1b. In practice the technique is implemented in a simplified form known as normal pulsed voltammetry ${ }^{1}$ where 
each chronoamperogram is sampled at a set time and one voltammogram is produced. This approach was particularly powerful when combined with mercury drop electrodes since each chronoamperogram could be recorded from a fresh drop. ${ }^{1}$
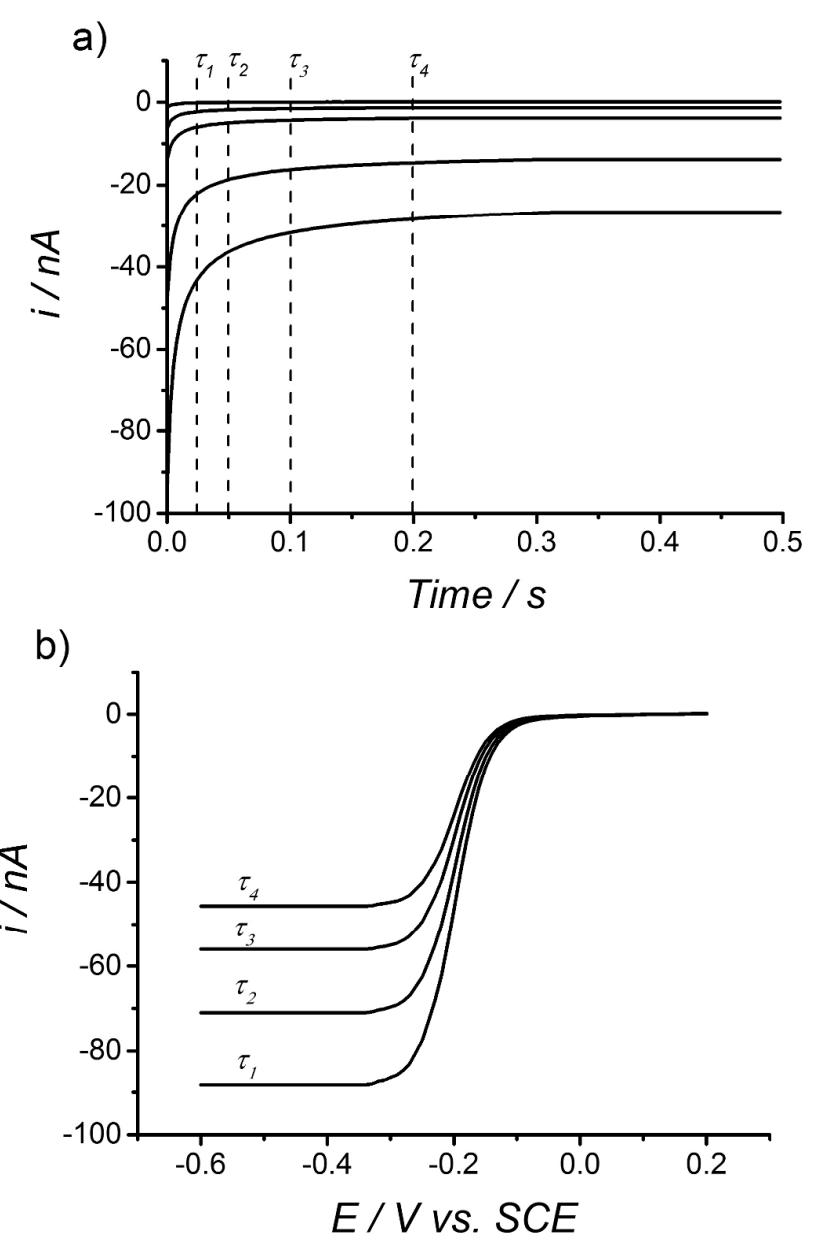

Figure 1: Plots showing the collected current vs. time transients (a) and the SCVs constructed from currents recorded at times $\tau_{1}, \tau_{2}, \tau_{3}$ and $\tau_{4}(\mathrm{~b})$.

In this study we demonstrate how applying SCV to microdisk electrodes offers new opportunities to exploit the unique properties of microelectrodes, particularly at short times. We are chiefly interested in harnessing and analyzing the Faradaic information available irrespective of the diffusion regime that controls mass transport to the electrode, i.e. irrespective of the timescale of the experiment. Furthermore we combine MSCV with a 
potential waveform carefully designed to renew the electrode surface before each potential step thus ensuring that every point on the sampled-current voltammogram corresponds to the same electrode surface state. This aspect is particularly important and appears to have been neglected as a key advantage of SCV at solid electrodes. With many metallic electrodes, e.g. Pt, the formation and removal of oxides or the adsorption and desorption of hydrogen create parallel redox processes which distort the Faradaic information at short times and make the analysis of chronoamperograms on the millisecond time scale particularly challenging unless some form of background subtraction is involved. In this respect it is worth noting that most short time amperometry at microelectrodes, typically carried out as high speed cyclic voltammetry, ${ }^{8-14}$ has been recorded in organic media where distortion from surface redox processes are much less pronounced than in aqueous media. It is also worth noting that while all the points on a given sampled-current voltammogram share the same history, SCVs for different sampling times reflect different electrode histories since the electrode surface evolves with time during a given potential step. Even though the electrode always starts from the same state after the pretreatment, data sampled at different sampling times correspond to different states of the electrode surface.

We first describe the conditioning waveform and normalizing procedure then validate the overall procedure with the reduction of $\mathrm{Ru}_{(}\left(\mathrm{NH}_{3}\right)_{6}{ }^{3+}$ on Pt microdisk electrodes. Ruthenium hexamine undergoes a rapid outer sphere one electron transfer process and was therefore chosen as a model system expected to produce diffusion controlled chronoamperograms on a sub-second timescale. Experiments were performed for different sampling times to validate the procedure over a range of mass transfer coefficients. Pt was chosen to illustrate the benefit of the conditioning procedure when operating with a metallic electrode known to promote oxide formation/stripping and hydrogen adsorption/desorption; we present results obtained with and without the conditioning waveform. In the remainder of the article we 
apply the methodology to assess the electron transfer kinetics for the reduction of $\mathrm{Fe}^{3+}$ on $\mathrm{Pt}$ microdisks in $\mathrm{HClO}_{4}$.

\section{EXPERIMENTAL CONDITIONS}

Materials: All electrochemical experiments were conducted in a two-electrode jacketed cell located inside a grounded Faraday cage and connected via plastic tubing to a water bath (Grant W14) set at $25^{\circ} \mathrm{C}$. To minimize interferences all water tubing was surrounded by a grounded metal wire mesh. A home-made $25 \mu \mathrm{m}$ diameter platinum microdisk electrode was used alongside a home-made saturated calomel reference electrode (SCE). The microdisk radius was determined from SEM measurements (gaseous secondary electrode detector, 0.6 Torr water vapor, $25 \mathrm{kV}$, with an XL30 ESEM environmental scanning electron microscope from FEI). Before each experiment the working electrode was polished for 20 min with 0.3 $\mu \mathrm{m}$ alumina powder on a polishing microcloth, both from Buehler, then cycled at $200 \mathrm{mV} \mathrm{s}^{-1}$ in the solution of interest until a stable voltammogram was seen. The electrodes were connected to a PC controlled PGSTAT101 Autolab, Ecochemie, for sampling times on the millisecond timescale or to a PGSTAT30 Autolab, Ecochemie, with the high speed ADC750 module enabled for sampling times on the microsecond timescale. Both potentiostats were operated with NOVA 1.10 also from Ecochemie. The solutions were prepared with ultrapure water (18 M $\Omega \mathrm{cm}$, Purlte, Burkert), $\mathrm{KCl}$ (99.6\%, Fisher), $\mathrm{Ru}\left(\mathrm{NH}_{3}\right)_{6} \mathrm{Cl}_{3}(98 \%$, Aldrich), $\mathrm{Fe}\left(\mathrm{ClO}_{4}\right)_{3}\left(99 \%\right.$, Aldrich) and $\mathrm{HClO}_{4}(70 \%$, Fluka). Oxygen was removed by purging with humid Ar (Pureshield, BOC) for 30 min. All gases were scrubbed and humidified by passing through a Drechsel bottle containing the same solution as in the cell. To avoid uptake of oxygen the gas line was made of glass. All glassware was soaked overnight in 5\% Decon 90 (BHD) and rinsed several times with pure water before use.

Electroanalytical methodology: A NOVA 1.10 procedure was written to automate the electrode pretreatment and collection of MSCVs. The electrode was first electrochemically 
cleaned by sweeping between upper and lower cleaning potentials at $500 \mathrm{mV} \mathrm{s}^{-1}$ six times. The potential was held for $10 \mathrm{~s}$ at the previously determined open circuit potential (OCP), then stepped to the potential of interest along the redox wave and held for $0.5 \mathrm{~s}$ during which the chronoamperometric response was recorded. The potential was then returned to its open circuit value for another $10 \mathrm{~s}$. This potential waveform, Figure 2, was then repeated, each time decreasing the step potential by $0.01 \mathrm{~V}$. A second NOVA procedure collated the transients and saved them as a text file while a third NOVA procedure reorganized the current transients collected at multiple potentials into sampled-current voltammograms, one for each sampling time.

Potentiostatic pretreatment: The conditioning waveform shown in Figure 2 was adapted from a potential waveform previously designed to pretreat Pt microdisk oxygen sensors. ${ }^{15}$ Briefly the upper cleaning potential was set in the foot of Pt oxide formation to promote oxidation of the Pt surface without increasing the surface roughness while the lower cleaning potential was set to promote the reduction of the Pt oxide and the adsorption of hydrogen. The rest potential was always set to the OCP to ensure zero current before the potential step. The directions of the potential sweeps were chosen to ensure the electrode was first oxidized then reduced. The end of the waveform was chosen so that the electrode would return to the OCP on a positive sweep having stripped the hydrogen from the surface. This guaranteed that the current recorded during the potential step did not include a Faradaic contribution from oxide reduction. As will be shown below this carefully designed waveform turned out to be crucially important to acquire purely diffusion controlled current with the Pt microdisks on a sub-second timescale. 


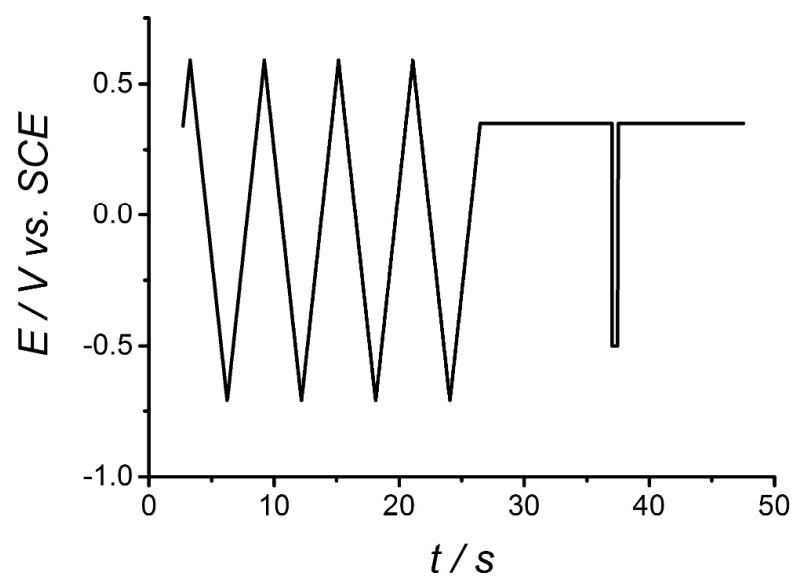

Figure 2: Potential waveform used to pretreat (sweeps) the microelectrode and acquire (step) the chronoamperometric data before reconstructing the sampled current voltammograms. This sequence was repeated, each time decreasing the step potential by $0.01 \mathrm{~V}$ from the open circuit potential to the plateau for the diffusion controlled reduction of interest.

\section{RESULTS AND DISCUSSION}

Normalization of the transients: Since the magnitude of the current depends on the time at which it is sampled all currents were normalized to facilitate comparison of MSCVs sampled at different times and to allow comparison of the MSCVs with the conventional steady state voltammogram obtained by linear sweep voltammetry at low sweep rate. Normalization was achieved by dividing each experimental current with the theoretical diffusion controlled current expected at a microdisk electrode of identical radius, for the same bulk concentration of redox species and importantly for the same time after the potential jump. This theoretical value was calculated using equation (1):

$I_{\text {theo }}^{\text {diff }}(\tau)=\pi n F D c^{\infty} a f\left(D \tau / a^{2}\right)$

where $n, F, D, c^{\infty}, a, \tau$ are respectively the number of electrons, Faraday's constant, bulk concentration, diffusion coefficient, electrode radius, time at which the current is considered 
and $f$ is a function proposed by Mahon and Oldham ${ }^{16}$ which accounts for the time dependence of the total flux to the microdisk:

$f(\sigma)=\left\{\begin{array}{ll}\frac{1}{\sqrt{\pi \sigma}}+1+\sqrt{\frac{\sigma}{4 \pi}}-\frac{3 \sigma}{25}+\frac{3 \sigma^{3 / 2}}{226} & \sigma \leq 1.281 \\ \frac{4}{\pi}+\frac{8}{\sqrt{\pi^{5} \sigma}}+\frac{25 \sigma^{-3 / 2}}{2792}-\frac{\sigma^{-5 / 2}}{3880}-\frac{\sigma^{-7 / 2}}{4500} & \sigma \geq 1.281\end{array}\right\}$

where $\sigma=D \tau / a^{2}$ is the dimensionless time.

Dividing the experimental current, $I_{\text {exp }}(\tau)$, by $I_{\text {theo }}^{\text {diff }}(\tau)$ (calculated with $n=1, D, c^{\infty}$ and $a$ set to known values and $\tau$ to the sampling time) produces a normalized current akin to an apparent number of electrons, $n_{a p p}$. Repeating this normalization for all potentials along the redox wave produces a normalized sampled-current voltammogram while repeating this normalization for multiple sampling times along the chronoamperograms for all the potentials considered produces a family of normalized MSCVs. Since the normalization removes the time dependence of the current, all normalized MSCVs should fall on top of each other provided the redox process is diffusion controlled.

This was verified using the reduction of $\mathrm{Ru}\left(\mathrm{NH}_{3}\right)_{6}{ }^{3+}$ as a model system. Employing the waveform described in Figure 2, chronoamperograms were recorded every $10 \mathrm{mV}$ from +0.2 to $-0.6 \mathrm{~V}$ vs. SCE, and sampled at set times after the potential step. The resulting sampled current voltammograms, Figure 3a, have the characteristic sigmoidal shape of steady state voltammograms as if they had been recorded under fixed mass transfer conditions, e.g. with a rotating disk electrode. As expected the corresponding limiting currents increase with shorter sampling times thus revealing the time dependence of the underlying process. In contrast, for the MSCVs recorded at long sampling times the limiting current converges towards the steady state limiting current normally obtained by linear sweep voltammetry at low scan rate, i.e. to that obtained under hemispherical diffusion. To compare the curves each experimental 
current value was then normalized with the theoretical current calculated for the relevant sampling time using equation (1) taking $n=1, D=8.4 \times 10^{-6} \mathrm{~cm}^{2} \mathrm{~s}^{-1}, c^{\infty}=5 \mathrm{mM}$ and $a=12.85 \mu \mathrm{m}$. $D$ was previously determined from a plot of the mass transport limited current recorded with microelectrodes of varying sizes, against the corresponding microelectrode radius, where the gradient was equal to $4 n F D c^{\infty}$.

Down to $10 \mathrm{~ms}$ sampling time the corresponding normalized MSCVs, Figure 3b, follow a unique sigmoidal curve thereby indicating that from $10 \mathrm{~ms}$ onwards the current is completely controlled by diffusion. The agreement between the normalized MSCVs is remarkable over the whole potential range except for the two shortest sampling times. While the currents sampled at $5 \mathrm{~ms}$ are a few percent smaller than the expected diffusion controlled values they appear somewhat noisier. This trend worsens with the currents sampled at $2.5 \mathrm{~ms}$, the shortest sampling time available with the PGSTAT101 potentiostat, as the currents are circa $10 \%$ smaller than expected and suffer the worse reproducibility. 


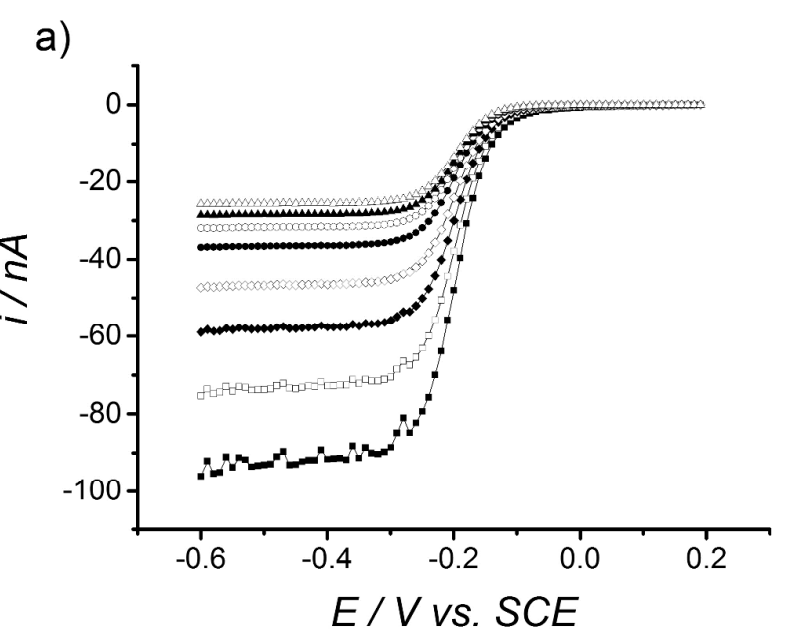

b)

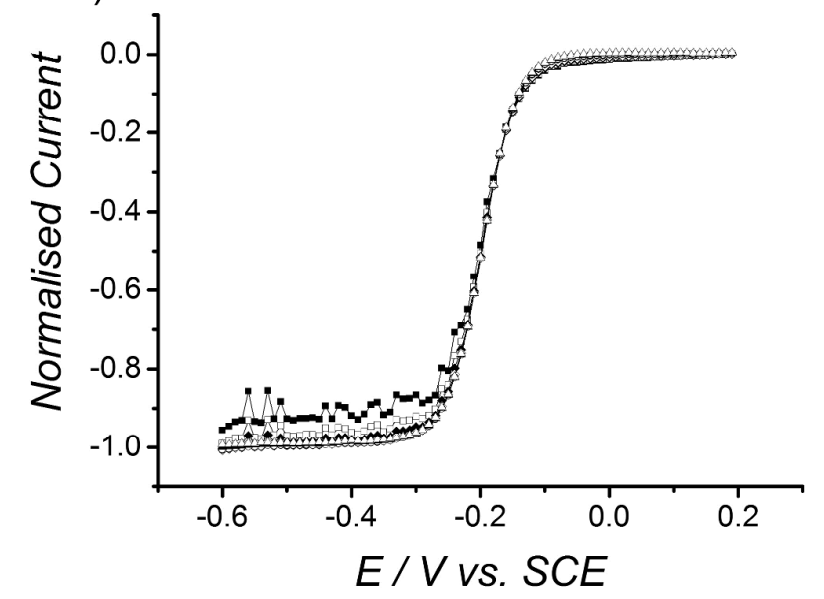

Figure 3: (a) MSCVs reconstructed from a series of chronoamperograms recorded in $\mathrm{Ar}$ purged $5 \mathrm{mM} \mathrm{Ru}\left(\mathrm{NH}_{3}\right)_{6} \mathrm{Cl}_{3}$ and $0.5 \mathrm{M} \mathrm{KCl}$ with a $25 \mu \mathrm{m}$ diameter Pt microdisk subjected to the waveform from Figure 2. For each potential the experimental currents were recorded every $2.5 \mathrm{~ms}$ using the high stability mode; the resulting transients were then sampled at 2.5 $\mathrm{ms}(\bullet), 5 \mathrm{~ms}(\square), 10 \mathrm{~ms}(\bullet), 20 \mathrm{~ms}(\diamond), 50 \mathrm{~ms}(\bullet), 100 \mathrm{~ms}(\circ), 200 \mathrm{~ms}(\boldsymbol{\Delta})$ and $500 \mathrm{~ms}(\Delta)$ after the potential step. (b) Corresponding normalized MSCVs obtained using equation (1) taking $n=1, D=8.4 \times 10^{-6} \mathrm{~cm}^{2} \mathrm{~s}^{-1}$ and $a=12.85 \mu \mathrm{m}$.

Influence of the acquisition conditions: The importance of the data acquisition conditions was demonstrated by repeating the construction of normalized MSCVs using 
chronoamperograms recorded with different acquisition procedures. In NOVA, as in many modern electrochemical systems, current acquisition at short timescales can be made more accurate by using the 'high speed' mode rather than the 'high stability' mode. Figure 4 shows a noticeable improvement of the normalized current when acquiring data with the high speed mode. Further improvement was obtained when using a faster potentiostat, PGSTAT30, to shorten the acquisition interval between data points. Chronoamperograms recorded with 10 $\mu$ s intervals yield a normalized MSCV with a plateau equal to unity. While a fast potentiostat operating under high speed mode ensures current transients are unaffected by the response time of the device these conditions do not allow the recording of long transients extending to the steady state regime as the number of data points acquired with the high speed mode is generally limited by the size of a memory buffer on the high speed ADC card. A compromise must therefore be found so as to get chronoamperograms undistorted at short times but long enough to show MSCVs converging towards the steady state regime. Guidelines for the choice of sampling times are considered in the Discussion section below. 


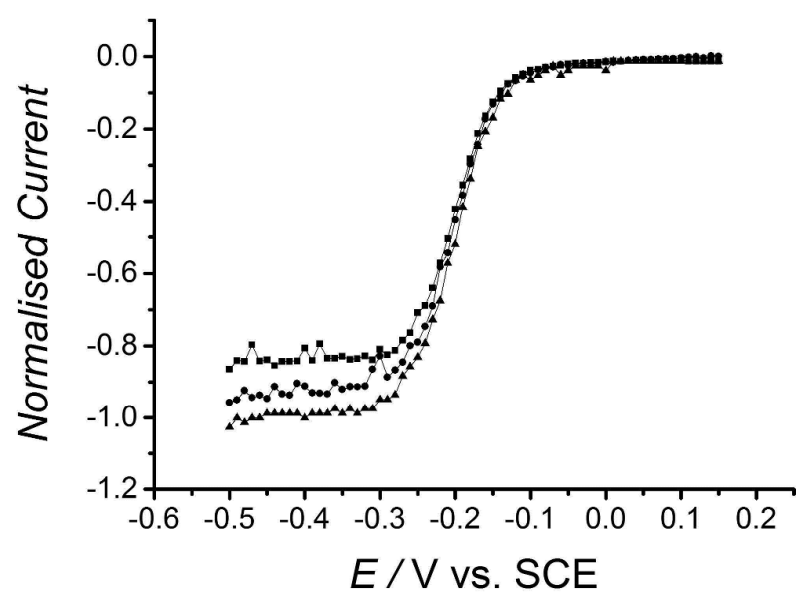

Figure 4: MSCVs reconstructed for the $2.5 \mathrm{~ms}$ sampling time from a series of chronoamperograms recorded in Ar purged $5 \mathrm{mM} \mathrm{Ru}\left(\mathrm{NH}_{3}\right)_{6} \mathrm{Cl}_{3}$ and $0.5 \mathrm{M} \mathrm{KCl}$ with a $25 \mu \mathrm{m}$ diameter Pt microdisk subjected to the waveform from Figure 2. The experimental currents were recorded every $2.5 \mathrm{~ms}$ under high stability ( $\bullet$ ) and high speed conditions $(\bullet)$ with the PGSTAT101, and every $10 \mu$ s under high speed conditions $(\boldsymbol{\Delta})$ with the PGSTAT30. Experimental currents were normalized with theoretical currents from equation 1 taking $n=1, D=8.4 \times 10^{-6} \mathrm{~cm}^{2} \mathrm{~s}^{-1}$ and $a=12.85 \mu \mathrm{m}$.

By converting the current-potential data recorded at different timescales into a single sigmoidal curve the normalization provides a very simple way of comparing and analyzing the data. This is particularly useful for microelectrodes because cyclic voltammograms change shape as the mass transport regime evolves from planar to hemispherical diffusion. ${ }^{17}$ As will be shown below, the approach is also very sensitive to slight deviations of the chronoamperometric response from the diffusion controlled behavior. At short sampling times such distortions may reflect instrumental or electrochemical artefacts, e.g. the response time of a slow current follower operated under high stability conditions as seen above or the contribution from double layer charging for large or porous electrodes. At long sampling times distortions may reflect the effect of natural convection on the limiting current. In the 
following sections we show that deviations from the diffusion controlled response also reflect complications from parallel adsorption/desorption processes or from heterogeneous kinetic limitations.

Role of the conditioning waveform: Chronoamperograms recorded without applying the conditioning waveform before each potential step produce normalized MSCVs which dramatically deviate from the unique sigmoidal current-potential relationship, particularly for short sampling times, Figure 5. The normalized MSCVs still follow a sigmoidal function but the currents are larger than the diffusion controlled response and systematically increase with shorter sampling times. This deviation was not observed when the electrode was preconditioned before each step, Figure 3b, so non-Faradaic processes such as double layer charging can be ruled out. The extra current therefore reflects the contribution from surface bound redox processes such as the reduction of Pt oxides. The adsorption of hydrogen could also affect the current but only at potentials below $-0.5 \mathrm{~V}$ because with the same electrolyte but no ruthenium hexamine the first peak for the adsorption of hydrogen on the Pt microdisk occurs at $-0.5 \mathrm{~V}$ vs. SCE on a cyclic voltammogram recorded at $200 \mathrm{mV} \mathrm{s}^{-1}$. Hence the rise of the sigmoidal curve and the beginning of the plateau are only affected by Pt oxide reduction if the waveform is not applied. As described previously the conditioning waveform was carefully designed to ensure every point on the sampled-current voltammogram was recorded in identical conditions and to remove the Faradaic contribution from surface-bound redox processes. The cleaning potentials and sweep directions guarantee that the electrode surface is first oxidized then reduced; the last conditioning sweep in particular guarantees that the open circuit potential is reached after having reduced the oxide and desorbed all hydrogen. Together Figure 5 and Figure 3 illustrate the dramatic improvements the conditioning waveform made to the current at short sampling times. To assess whether similar 
improvements could be obtained by background subtraction, MSCV experiments were carried out without the conditioning waveform and without $\mathrm{Ru}\left(\mathrm{NH}_{3}\right)_{6} \mathrm{Cl}_{3}$ to produce a background sampled-current voltammogram for each sampling time. Prior to normalization these were subtracted from the corresponding MSCVs recorded in presence of ruthenium hexamine. The background subtraction approach was not only far more cumbersome, it required two solutions and took at least twice longer, but also turned out to be far less successful than the conditioning waveform as the resulting background subtracted normalized MSCVs, not shown, were worse, particularly at short sampling times, than without background subtraction and never fitted a unique sigmoidal response. The ability to condition the electrode before each potential step is therefore a key advantage of sampled-current voltammetry. This had been exploited when normal pulse voltammetry was applied to dropping mercury electrodes since each step was recorded with a fresh drop. With programmable computerized instrumentation any form of electrochemical waveform can be implemented within the SCV protocol to refresh the surface of solid electrodes before each step. To our knowledge this unique advantage of SCV has been overlooked. In the following section we exploit theoretical treatments developed to analyze steady state voltammograms at microelectrodes to extract electron transfer kinetic information from the MSCVs. 


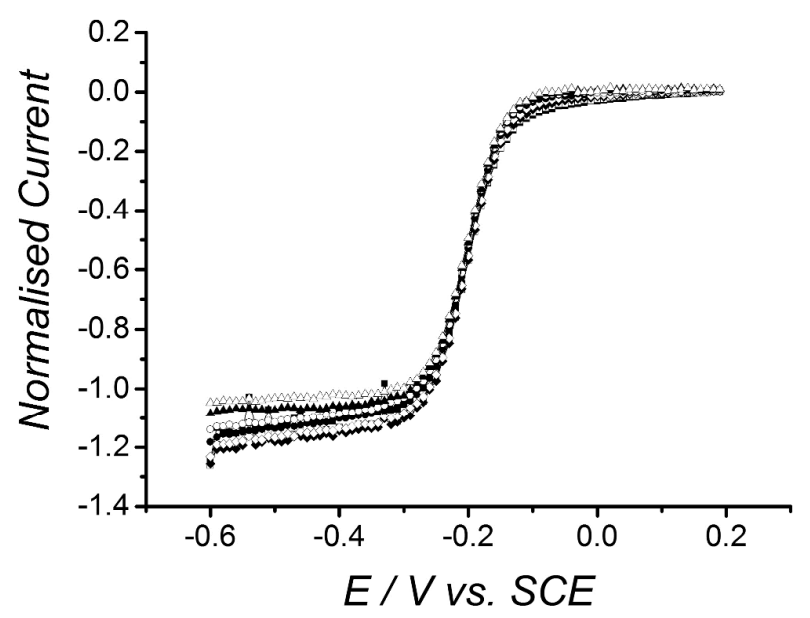

Figure 5: Normalized MSCVs recorded without the preconditioning waveform shown in Figure 2. Symbols and other experimental details are as in Figure 3.

Kinetic information: $\mathrm{MSCVs}$ were recorded for the reduction of $5 \mathrm{mM} \mathrm{Fe}\left(\mathrm{ClO}_{4}\right)_{3}$ in $0.5 \mathrm{M}$ $\mathrm{HClO}_{4}$ using a $25 \mu \mathrm{m}$ diameter $\mathrm{Pt}$ microdisk. The diffusion coefficient for $\mathrm{Fe}^{3+}$ in this solution, $6.3 \times 10^{-6} \mathrm{~cm}^{2} \mathrm{~s}^{-1}$, was determined from a plot of limiting currents against microdisk radii for different microdisks. The theoretical diffusion controlled current was then calculated with equation 1 and used to normalize the experimental currents. Figure 6 shows that, irrespective of sampling times, all normalized currents equal one for potentials below $0.1 \mathrm{~V}$ vs. SCE; this therefore indicates that the reduction of $\mathrm{Fe}^{3+}$ is controlled by diffusion below this potential. At more positive potentials the normalized MSCVs are affected by the sampling time with MSCVs obtained at long times giving a noticeably steeper slope than those obtained at short times. For example at $500 \mathrm{~ms}$ the sigmoidal curve is steep and akin to a reversible wave but with shorter sampling times the curve becomes increasingly drawn to more negative potentials; this is indicative of increasing electron transfer kinetics effects. Figure 6 illustrates a key property of the normalization which reveals kinetic limitations by removing the influence of the different diffusion regimes on the shape of the current-potential curve. To our knowledge this approach has never been applied to microdisk electrodes and no 
analytical expression exists to extract the kinetic information clearly displayed in the normalized MSCVs. The methods reported to extract kinetic parameters from the shape of quasi-reversible steady state voltammograms at microelectrodes ${ }^{18-20}$ are not suitable because they apply to steady state mass transfer conditions and it is known that care must be exercised when extracting kinetic information from voltammetric and chronoamperometric responses at microdisks under conditions intermediate between planar and hemispherical diffusion. ${ }^{21-23}$ Therefore to derive $E^{0}, k^{0}$ and $\alpha$, respectively the standard potential, standard heterogeneous rate constant and transfer coefficient, an expression developed by Oldham et al. ${ }^{19}$ to extract kinetic information from voltammograms arising from steady state hemispherical diffusion at microdisk electrodes was modified to account for the time dependence of the mass transport. To account for the influence of the different diffusion regimes the steady state diffusion controlled current in their original expression was replaced with $I_{\text {theo }}^{\text {diff }}$ from equation 1 and the steady state mass transfer coefficients for $\mathrm{O}$ and $\mathrm{R}$ were replaced by time dependent mass transfer coefficients also derived from equation 1 and given by

$k_{m, \mathrm{i}}=\frac{D_{\mathrm{i}}}{a} f\left(D_{\mathrm{i}} \tau / a^{2}\right)$

where subscript i represents the species of interest and $f$ is the function given in equation 2 . These modifications yield a new expression, equation 5, which predicts the dependence of the microdisk current on potential and time for quasi-reversible electron transfer kinetic conditions, i.e. the shape of the MSCVs affected by kinetic limitations.

$i_{\text {theo }}(E, \tau)=\frac{\pi n F D_{\mathrm{O}} c_{\mathrm{O}}^{\infty} a f\left(D_{\mathrm{O}} \tau / a^{2}\right)}{\theta}\left[1+\frac{\pi}{\kappa \theta}\left(\frac{2 \kappa \theta+3 \pi}{4 \kappa \theta+3 \pi^{2}}\right)\right]^{-1}$

where

$\kappa=\frac{k^{0} a}{D_{\mathrm{O}} f\left(D_{\mathrm{O}} \tau / a^{2}\right)} \exp \left\{\frac{-\alpha n F\left(E-E^{0}\right)}{R T}\right\}$

and

$\theta=1+\frac{D_{\mathrm{O}} f\left(D_{\mathrm{O}} \tau / a^{2}\right)}{D_{\mathrm{R}} f\left(D_{\mathrm{R}} \tau / a^{\wedge} 2\right)} \exp \left\{\frac{n F\left(E-E^{0}\right)}{R T}\right\}$ 


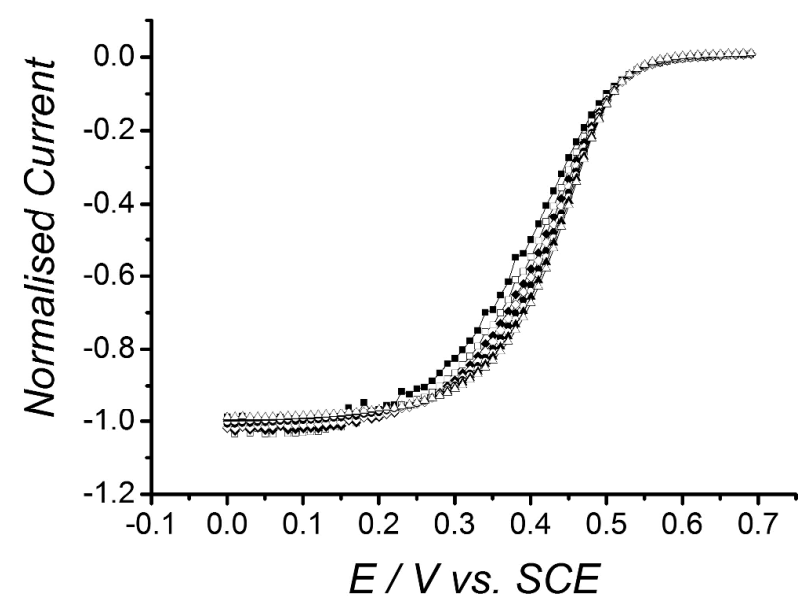

Figure 6: Normalized MSCVs recorded with a $25 \mu \mathrm{m}$ diameter Pt disk in Ar purged $5 \mathrm{mM}$ $\mathrm{Fe}\left(\mathrm{ClO}_{4}\right)_{3}$ and $0.5 \mathrm{M} \mathrm{HClO}_{4}$. The experimental current transients were sampled at $2.5 \mathrm{~ms}(\mathbf{\square})$, $5 \mathrm{~ms}(\square), 10 \mathrm{~ms}(\bullet), 20 \mathrm{~ms}(\diamond), 50 \mathrm{~ms}(\bullet), 100 \mathrm{~ms}(\odot), 200 \mathrm{~ms}(\boldsymbol{\Delta})$ and $500 \mathrm{~ms}(\Delta)$ after the potential step. Normalization was done with equation 1 taking $n=1, D=6.3 \times 10^{-6} \mathrm{~cm}^{2} \mathrm{~s}^{-}$ ${ }^{1}$ and $a=12.85 \mu \mathrm{m}$. The conditioning waveform shown in Figure 2 was used to prepare the electrode before each step.

The parameters $E^{0}, k^{0}$ and $\alpha$ were hence determined by nonlinear curve fitting of the experimental MSCVs to equation 5 using Origin 9.1. The above parameters were set as adjustable, whilst $c_{\mathrm{O}}^{\infty}, D_{\mathrm{O}}, D_{\mathrm{R}}$ and sampling time $\tau$ were fixed. Since $E^{0}, k^{0}$ and $\alpha$ are independent of the sampling time, five experimental MSCVs respectively recorded at $\tau=500,400,300,200$ and $100 \mathrm{~ms}$ were simultaneously fitted to equation 5 using the global fitting facility of Origin 9.1. The high quality of the fit $\left(\mathrm{R}^{2}=0.999\right)$ reached after a few iterations meant that different weightings (including a Gaussian distribution of weights around the slope of the sigmoidal) made a negligible difference to the extracted parameters. From the regression analysis $E^{0}=0.496 \mathrm{~V}$ vs. SCE, $k^{0}=0.008 \mathrm{~cm} \mathrm{~s}^{-1}$, and $\alpha=0.366$. These 
values are in good agreement with those previously derived from rotating disk electrode experiments, $k^{0}=0.009 \mathrm{~cm} \mathrm{~s}^{-1}$ and $0.008 \mathrm{~cm} \mathrm{~s}^{-1}$ with $\alpha=0.37 .{ }^{24,25}$ The quality of the fit of the experimental MSCVs to equation 5, irrespective of the sampling time, is clearly seen in Figure 7. A full discussion of the model presented in equation 5, along with its range of validity across varying sampling times and degrees of reversibility will be reported in a subsequent article, currently under development.

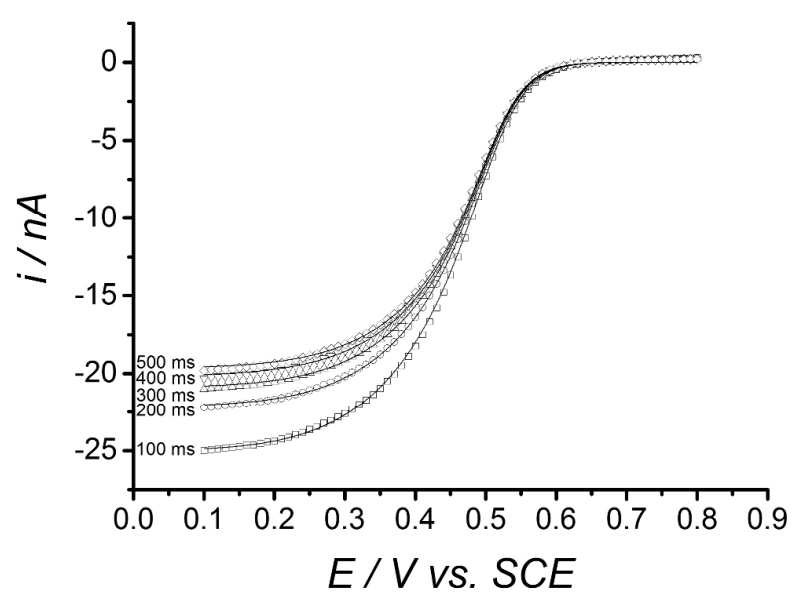

Figure 7: Experimental (hollow symbols) and theoretical (lines) MSCVs for a $25 \mu \mathrm{m}$ diameter Pt disk in Ar purged $5 \mathrm{mM} \mathrm{Fe}\left(\mathrm{ClO}_{4}\right)_{3}$ and $0.5 \mathrm{M} \mathrm{HClO}_{4}$. The experimental current transients were sampled at the times indicated against the MSCVs. The theoretical curves were produced by nonlinear curve fitting of the experimental data to equation 5 with $n=1$, $D_{O}=6.3 \times 10^{-6} \mathrm{~cm}^{2} \mathrm{~s}^{-1}, D_{R}=7.6 \times 10^{-6} \mathrm{~cm}^{2} \mathrm{~s}^{-1},{ }^{26}$ and $a=12.85 \mu \mathrm{m}$. The conditioning waveform shown in Figure 2 was used to prepare the electrode before each step.

To validate the approach and equation 5, COMSOL Multiphysics (for a description of the model see Supporting Information) was used to generate a number of theoretical current transients for the times and potentials considered in the experiments. Parameters $D_{O}, D_{R}, c, a$ and $\tau$ were set to match the values quoted above while $E^{0}, k^{0}$ and $\alpha$ were set to the values 
determined by nonlinear regression of the experimental data to equation 5 . Theoretical MSCVs were then constructed by sampling the simulated transients at the same times used for the experimental data. Good agreement between experimental and simulated MSCVs, Figure 8, validates the derived parameters and confirms the suitability of equation 5 .

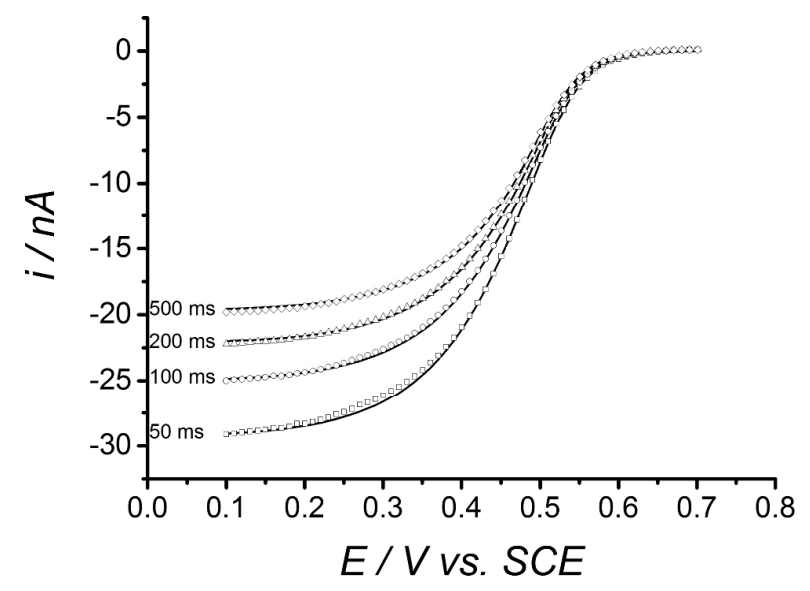

Figure 8: Comparison of the experimental MSCVs (hollow symbols) and COMSOL simulated MSCVs (-) for the reduction of $\mathrm{Fe}^{3+}$. Experimental conditions as in Figure 7. The simulation parameters were $n=1, D_{O}=6.3 \times 10^{-6} \mathrm{~cm}^{2} \mathrm{~s}^{-1}, D_{R}=7.6 \times 10^{-6} \mathrm{~cm}^{2} \mathrm{~s}^{-1}$, $a=12.85 \mu \mathrm{m}, E^{0}=0.496 \mathrm{~V}$ vs. SCE, $k^{0}=0.008 \mathrm{~cm} \mathrm{~s}^{-1}$ and $\alpha=0.366$.

Discussion: Thanks to their very small electroactive area microdisk electrodes allow the acquisition of amperometric data unaffected by double layer charging currents well below the millisecond timescale; ${ }^{14}$ e.g. in $0.5 \mathrm{M} \mathrm{NaCl} 95 \%$ of the charge and discharge of the double layer at a $25 \mu \mathrm{m}$ diameter disk occurs under $3 \mu$ s (see Supporting Information). Hence pseudo-steady state sampled-current voltammograms free from capacitive distortions can be reliably recorded on sub-millisecond timescales. With microdisks, voltammograms recorded at short times differ markedly from those recorded at long times because of the transition from planar to hemispherical diffusion and it is particularly difficult to compare and analyze the CVs recorded on different time scales. With the normalized MSCVs reported here it 
becomes possible to compare the microelectrode voltammograms at all timescales, i.e. for any diffusion regime.

The range of sampling times appropriate to record the MSCVs must be adjusted depending on experimental conditions such as the specifications of the potentiostat (e.g. acquisition rate, response time of current follower), the solution parameters (viscosity, concentration and diffusion coefficient of the redox species) and the microdisk radius. Since the normalization implies that diffusion is the only form of mass transport, the longest sampling time should follow the criteria reported by Amatore et al. ${ }^{27}$ so that the corresponding MSCV is not affected by natural convection. ${ }^{28,29}$ In contrast, the shortest sampling time should be sufficiently long to ensure the current is not affected by the response time of the current follower. Ideally the current should be recorded with a non-linear amplifier so as to exploit the whole dynamic range of the transient but since commercially available electrochemical workstations operate with linear amplifiers the current sensitivity should be low enough to avoid saturation of the current follower at short times and high enough to afford a reliable measure of the long-time current. If the current sensitivity and acquisition rate chosen force the amplifier to saturate at short times, the shortest sampling time should be long enough to ensure that the amplifier has recovered from the saturation (recovery times vary between Op Amps and can be longer than the duration of the saturation). In the experiments presented here the current sensitivity was such that the amplifier never saturated.

The MSCV timescale can be compared with that of a conventional voltammogram as the scan rate needed to achieve the same conditions under linear sweep voltammetry can be expressed through equation $8^{1}$

$v=\frac{R T}{F \tau}$

For the sampling times used in this work, 500 to $2.5 \mathrm{~ms}$, the equivalent sweep rates range between $50 \mathrm{mV} \mathrm{s}^{-1}$ and $10 \mathrm{~V} \mathrm{~s}^{-1}$ while sampling at $1.33 \mu \mathrm{s}$, the shortest sampling time 
available with the high speed ADC750 module of the Autolab, equates to a $19.3 \mathrm{kV} \mathrm{s}^{-1}$ scan rate. The MSCV procedure therefore offers a wide range of apparent sweep rates, simply by sampling the same data set at different times. This approach should be particularly useful to determine the electron transfer kinetics in cases where the diffusion regime is affected by both planar and quasi-hemispherical diffusion, as in viscous media such as room temperature ionic liquids. $^{21}$

Although only used to qualitatively analyze the MSCVs shape, the normalization procedure requires the precise knowledge of $D, c$ and $a$, and a great deal of experimental care is needed to ensure these parameters stay constant throughout the course of the experiment, as data collection is fairly time consuming; e.g. the MSCVs shown in Figure 3 took $4000 \mathrm{~s}$ to record (80 potential steps taking $50 \mathrm{~s}$ each as shown in Figure 2). Temperature control with a thermostatically controlled water bath and precise measurement of $a, D$ and $c$ are therefore essential. Inclusion of the cleaning waveform is also necessary for high quality data but this greatly increases data collection time. However this process can be readily automated with standard electrochemical software.

The MSCVs recorded at short times have large currents and it is worth considering whether their shape is affected by Ohmic distortion. When sampling at $2.5 \mathrm{~ms}$, the diffusion controlled current for a step in the plateau region is approximately $98.7 \mathrm{nA}$ for the conditions employed in Figure 4. The corresponding uncompensated solution resistance (estimated using equation 3 in SI) and $i R$ drop are respectively $\sim 3078 \Omega$ and $\sim 0.3 \mathrm{mV}$. When sampling at 10 $\mu \mathrm{s}$, the diffusion controlled current rises to $1.3 \mu \mathrm{A}$ and the $i R$ drop to $4 \mathrm{mV}$. In both cases the $i R$ drop is sufficiently small to consider the SCV as unaffected by Ohmic distortion. Obviously more dilute electrolytes would yield larger $i R$ drops and distort the MSCVs. This should be minimized by selecting smaller microdisks. 


\section{CONCLUSIONS}

In this work we combined the unique properties of microdisk electrodes with sampledcurrent voltammetry, an approach apparently never previously undertaken, and reported a simple protocol to analyze and compare the sampled-current voltammograms irrespective of timescale and diffusion regime. This is particularly useful for microelectrodes where conventional cyclic voltammograms dramatically change shape with the diffusion regime when varying the sweep rate. We also combined SCV at Pt microdisks with a conditioning waveform to produce voltammograms where each data point was recorded with the same electrode history and demonstrated that the waveform was crucial to obtaining reliable sampled-current voltammograms below $100 \mathrm{~ms}$. We derived a simple analytical expression, equation 5, which accounts for the diffusion regime and kinetic limitations and showed that the microdisk sampled current voltammograms were easily analyzed to extract electron transfer kinetic parameters. For the reduction of $\mathrm{Fe}^{3+}$ in $0.5 \mathrm{M} \mathrm{HClO}_{4}$ this technique gave $\alpha=$ $0.37, k^{0}=0.008 \mathrm{~cm} \mathrm{~s}^{-1}$ and $E^{0}=0.496 \mathrm{~V}$ vs. SCE. The methodology reported here is novel and opens up new opportunities to exploit the unique properties of microelectrodes, in particular at short times; furthermore it is easily implemented on existing computer controlled electrochemical workstations as a new electroanalytical method.

\section{ASSOCIATED CONTENT}

\section{Supporting Information}

Additional material as described in text. This material is available free of charge via the Internet at http://pubs.acs.org.

\section{AUTHOR INFORMATION}

\section{Corresponding Author}

*gd@soton.ac.uk 


\title{
Notes
}

The authors declare no competing financial interest.

\section{ACKNOWLEDGMENT}

SCP acknowledges the support of the Faculty of Natural and Environmental Sciences, University of Southampton, LMAS acknowledges a scholarship from the government of Oman and GD acknowledges a scholarship from the Hanse Wissenschaftkoleg, Delmenhorst, Germany.

\begin{abstract}
ABBREVIATIONS
SCV, sampled current voltammetry; MSCV, microdisk sampled current voltammetry; SCE, saturated calomel electrode.
\end{abstract}

\section{REFERENCES}

(1) Bard, A. J.; Faulkner, L. R. Electrochemical methods: Fundamentals and Applications, 2nd ed.; Wiley: New York, 2001.

(2) Mirkin, M. V. In Handbook of Electrochemistry, Zoski, C. G., Ed.; Elsevier, 2007, pp 639-660.

(3) Murphy, M. M.; Odea, J. J.; Osteryoung, J. Anal. Chem. 1991, 63, 2743-2750.

(4) Osteryoung, J. G.; Osteryoung, R. A. Anal. Chem. 1985, 57, A101-\&.

(5) Odea, J. J.; Osteryoung, J.; Osteryoung, R. A. Anal. Chem. 1981, 53, 695-701.

(6) Odea, J.; Wojciechowski, M.; Osteryoung, J.; Aoki, K. Anal. Chem. 1985, 57, 954-955.

(7) Osteryoung, J. Acc. Chem. Res. 1993, 26, 77-83.

(8) Andrieux, C. P.; Hapiot, P.; Saveant, J. M. J. Phys. Chem. 1988, 92, 5987-5992.

(9) Fortgang, P.; Amatore, C.; Maisonhaute, E.; Schöllhorn, B. Electrochem. Commun. 2010, 12, 897-900.

(10) Howell, J. O.; Wightman, R. M. Anal. Chem. 1984, 56, 524-529.

(11) Howell, J. O.; Wightman, R. M. J. Phys. Chem. 1984, 88, 3915-3918.

(12) Andrieux, C. P.; Garreau, D.; Hapiot, P.; Saveant, J. M. Journal of Electroanalytical Chemistry 1988, 248, 447-450.

(13) Andrieux, C. P.; Hapiot, P.; Saveant, J. M. J. Phys. Chem. 1988, 92, 5992-5995.

(14) Wightman, R. M.; Wipf, D. O. Acc. Chem. Res. 1990, 23, 64-70.

(15) Sosna, M.; Denuault, G.; Pascal, R. W.; Prien, R. D.; Mowlem, M. Sensors and Actuators B: Chemical 2007, 123, 344-351.

(16) Mahon, P. J.; Oldham, K. B. Anal. Chem. 2005, 77, 6100-6101.

(17) Forster, R. J.; Keyes, T. E. In Handbook of Electrochemistry, Zoski, C. G., Ed.; Elsevier: Amsterdam, 2007, pp 155-188.

(18) Oldham, K. B.; Zoski, C. G. Journal of Electroanalytical Chemistry 1988, 256, 11-19.

(19) Oldham, K. B.; Myland, J. C.; Zoski, C. G.; Bond, A. M. Journal of Electroanalytical Chemistry 1989, 270, 79-101. 
(20) Mirkin, M. V.; Bard, A. J. Anal. Chem. 1992, 64, 2293-2302.

(21) Barnes, A. S.; Rogers, E. I.; Streeter, I.; Aldous, L.; Hardacre, C.; Compton, R. G. The Journal of Physical Chemistry B 2008, 112, 7560-7565.

(22) Barnes, A. S.; Streeter, I.; Compton, R. G. Journal of Electroanalytical Chemistry 2008, 623, 129-133.

(23) Belding, S. R.; Rogers, E. I.; Compton, R. G. J. Phys. Chem. C 2009, 113, 4202-4207.

(24) Suzuki, J. Bulletin of the Chemical Society of Japan 1970, 43, 755-\&.

(25) Angell, D. H.; Dickinso.T. Journal of Electroanalytical Chemistry 1972, 35, 55-\&.

(26) Galus, Z.; Golas, J.; Osteryoung, J. The Journal of Physical Chemistry 1988, 92, 1103 1107.

(27) Amatore, C.; Pebay, C.; Thouin, L.; Wang, A. F.; Warkocz, J. S. Anal. Chem. 2010, 82, 6933-6939.

(28) Gao, X. P.; Lee, J.; White, H. S. Anal. Chem. 1995, 67, 1541-1545.

(29) Amatore, C.; Szunerits, S.; Thouin, L.; Warkocz, J. S. Journal of Electroanalytical Chemistry 2001, 500, 62-70.

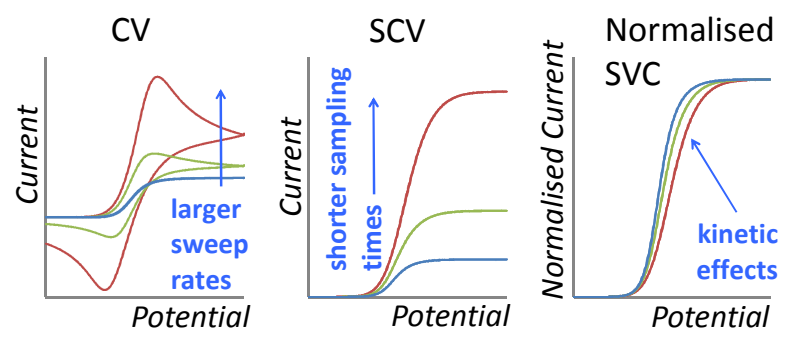

For Table of Contents Only 